\title{
Potential protective effect of hesperidin on hypoxia/reoxygenation-induced hepatocyte injury
}

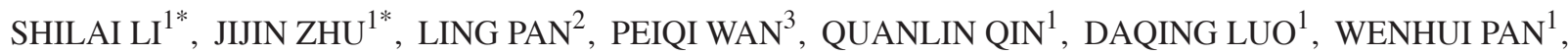 \\ YUQING WEI $^{1}$, YANSONG XU ${ }^{1}$, LIMING SHANG $^{4}$ and XINPING YE ${ }^{4}$
}

Departments of ${ }^{1}$ Emergency, ${ }^{2}$ Nephrology, ${ }^{3}$ Infectious Diseases and ${ }^{4}$ Hepatobiliary Surgery,

The First Affiliated Hospital of Guangxi Medical University, Nanning, Guangxi 530021, P.R. China

Received December 15, 2020; Accepted April 30, 2021

DOI: $10.3892 / \mathrm{etm} .2021 .10196$

\begin{abstract}
Hesperidin (HDN) has been reported to have hydrogen radical- and hydrogen peroxide-removal activities and to serve an antioxidant role in biological systems. However, whether HDN protects hepatocytes (HCs) against hypoxia/reoxygenation (H/R)-induced injury remains unknown. The present study aimed to explore the role of $\mathrm{HDN}$ in H/R-induced injury. HCs were isolated and cultured under $\mathrm{H} / \mathrm{R}$ conditions with or without HDN treatment. HC damage was markedly induced under $H / R$, as indicated by cell viability, supernatant lactate dehydrogenase levels and alanine aminotransferase levels; however, HDN treatment significantly reversed HC injury. Oxidative stress markers (malondialdehyde, superoxide dismutase, glutathioneand reactive oxygen species) were increased markedly during $H / R$ in HCs; however, this effect was significantly attenuated after exposure to HDN. Compared with those of the control group, the mRNA expression levels of IL-6 and TNF- $\alpha$ in HCs and the concentrations of IL- 6 and TNF- $\alpha$ in the supernatants increased significantly following $\mathrm{H} / \mathrm{R}$, and HDN significantly ameliorated these effects. Western blotting demonstrated that microtubule-associated protein 1 light chain $3 \alpha$ (MAP1LC3A, also known as LC3) and Beclin-1 protein expression levels increased, while sequestosome 1 levels decreased during $\mathrm{H} / \mathrm{R}$ following exposure to HDN. The number of GFP-LC3 puncta in HCs following exposure to HDN was increased compared with that observed in HCs without HDN exposure under the $\mathrm{H} / \mathrm{R}$ conditions after bafilomycin A1 treatment. In summary,
\end{abstract}

Correspondence to: Dr Liming Shang or Dr Xinping Ye, Department of Hepatobiliary Surgery, The First Affiliated Hospital of Guangxi Medical University, 6 Shuangyong Road, Nanning, Guangxi 530021, P.R. China

E-mail: shangliming@gxmu.edu.cn

E-mail: yexinping@gxmu.edu.cn

*Contributed equally

Key words: hesperidin, hypoxia/reoxygenation, hepatocyte, oxidative stress, autophagy the present study demonstrated that HDN attenuated $\mathrm{HC}$ oxidative stress and inflammatory responses while enhancing autophagy during H/R. HDN may have a potential protective effect on HCs during H/R-induced injury.

\section{Introduction}

Ischemia/reperfusion (I/R) injury remains the major cause of liver injury during major liver resection and transplantation $(1,2)$. Oxidative stress is supposed to be the major initiating mechanism $(3,4)$. In recent decades, increasing studiesintended to illuminate the molecular and pathological mechanisms in liver I/R injury have been reported; however, only a partial understanding of these processes is currently available $(1,2)$. $\mathrm{I} / \mathrm{R}$ injury is a critical condition caused by interrupted blood supply, and potentially, it is caused by hypoxia/reoxygenation (H/R) (5-7). The in vitro $\mathrm{H} / \mathrm{R}$-induced injury model is the best model available with which to mimic and study the in vivo I/R injury (5-7). Excessive production of reactive oxygen species (ROS) along with a dramatic decrease in antioxidant defenses is observed during $\mathrm{H} / \mathrm{R}(3,8)$. Oxidative damage and inflammatory responses are responsible for I/R injury and H/R injury $(3,8)$. Thus, these attributes could serve as therapeutic targets for the prevention and treatment of I/R injury.

Hesperidin (HDN) is a bioflavonoid with antibacterial, anti-inflammatory, and antioxidant effects (9-12). It was reported that HDN has hydrogen radical- and hydrogen peroxide-removal activities and serves an antioxidant role in biological systems $(9,10)$. We and others have revealed that HDN has a protective effect against I/R injury (13-17). It is also reported that $\mathrm{HDN}$ protects against $\mathrm{H} / \mathrm{R}$ injury in vitro in rat cardiomyocytes and a human first-trimester trophoblast cell line $(18,19)$. However, whether HDN protects hepatocytes (HCs) against H/R-induced injury remains unknown.

The present study hypothesized that HDN may ameliorate $\mathrm{H} / \mathrm{R}$-induced injury in $\mathrm{HCs}$ in vitro. $\mathrm{HCs}$ were isolated and cultured under H/R conditions with or without HDN exposure. The present study revealed that HDN ameliorated $\mathrm{H} / \mathrm{R}$-induced injury in HCs in vitro. Furthermore, the results demonstrated that HDN attenuated $\mathrm{HC}$ oxidative stress and inflammatory responses while enhancing autophagy during $\mathrm{H} / \mathrm{R}$. Thus, exposure to HDN may have a protective effect on HCs during H/R-induced injury. 


\section{Materials and methods}

Hepatocyte isolation and culture. A total of 3 male C57BL/6J WT mice aged 8-12 weeks and weighing 22-30 g were purchased from and housed in Guangxi Medical University Laboratory Animal Center (Guangxi, China). The mice were bred in a specific pathogen-free animal facility under controlled conditions at $19-23^{\circ} \mathrm{C}$ and $40-60 \%$ humidity with a 12-h dark/light cycle and had free access to food and water. HCs were isolated from mice as described previously $(17,20)$. Briefly, the mice wereeuthanized with 5\% isoflurane for $5 \mathrm{~min}$ in a plexiglass chamber, followed by bilateral thoracotomy for a secondary confirmation of death. Thenthe mice were perfused using an in situ collagenase (type VI; WorthingtonBiochemical Corporation) technique in vivo. HCs were separated from nonparenchymal cells and purified to $>99 \%$ purity with a viability $>95 \%$, as confirmed by trypan blue exclusion. HCs were cultured as described previously $(17,21)$. HCs $\left(1.5 \times 10^{5}\right.$ cells $\left./ \mathrm{ml}\right)$ were plated on gelatin-coated culture plates with collagen I (BD Pharmingen; BD Biosciences) in cell culture medium. The cell culture media contained Williams medium E (Invitrogen; Thermo Fisher Scientific, Inc.) with $10 \%$ calf serum (Thermo Fisher Scientific, Inc.), $15 \mathrm{mM}$ HEPES (Thermo Fisher Scientific, Inc.), $1 \mu \mathrm{M}$ insulin (Eli Lilly and Company), $2 \mathrm{mM}$ L-glutamine (Thermo Fisher Scientific, Inc.), penicillin $(100 \mathrm{U} / \mathrm{ml})$ and streptomycin $(100 \mathrm{U} / \mathrm{ml}$; Invitrogen; Thermo Fisher Scientific, Inc.). HCs were cultured overnight at $37^{\circ} \mathrm{C}$, and the culture medium was replaced with fresh medium before the experimental treatment. For H/R treatment, HCs were incubated under hypoxic conditions ( $1 \%$ oxygen for $10 \mathrm{~h}$ ) following reoxygenation (normoxic conditions for $8 \mathrm{~h}$ ). HCs were divided into the following groups: Control PBS group (HCs were subjected to normoxia with PBS, $18 \mathrm{~h}$ ), control HDN group (HCs were subjected to normoxia with $50 \mu \mathrm{g} / \mathrm{ml} \mathrm{HDN}, 18 \mathrm{~h}$ ), H/R PBS group (HCs were subjected to $\mathrm{H} / \mathrm{R}$ with $\mathrm{PBS}, \mathrm{H} / \mathrm{R}$ for 10 and $8 \mathrm{~h}$ as aforementioned) and $\mathrm{H} / \mathrm{R}$ HDN group (HCs were subjected to H/R with $50 \mu \mathrm{g} / \mathrm{ml} \mathrm{HDN}$, $\mathrm{H} / \mathrm{R}$ for 10 and $8 \mathrm{~h}$ as aforementioned).

Reagents. For western blotting, the autophagy antibody sample kit (cat. no. 4445; Cell Signaling Technology, Inc.; 1:1,000) was used, which targets the proteins microtubule-associated protein 1 light chain $3 \alpha$ (MAP1LC3A, also known as LC3), Beclin-1, and sequestosome 1 (SQSTM1, also known as P62). GAPDH (cat. no. ab8245; Abcam; 1:2,500) was used as the internal control. The goat anti-mouse secondary antibody (cat. no. 31430) and goat anti-rabbit secondary antibody (cat. no. 31460) (both at 1:20,000 dilution) were from Thermo Fisher Scientific, Inc. HDN (HPLC >98\%; cat. no. XW05202631) was obtained from Sinopharm Chemical Reagent Co., Ltd. The malondialdehyde assay kit (MDA; cat. no. A003-1), superoxide dismutase assay kit (SOD; cat. no. A001-1), glutathione assay kit (GSH; cat. no. A006-1), interleukin- 6 assay kit (IL-6; cat. no. H007) and tumor necrosis factor- $\alpha$ assay kit (TNF- $\alpha$; cat. no. H052) were purchased from Nanjing Jiancheng Bioengineering Institute.

Supernatant sample assays. Supernatant alanine aminotransferase (ALT) levels were measured using an ALT assay kit according to the manufacturer's instructions (cat. no. C009-2-1;
Nanjing Jiancheng Institute of Biotechnology) and analyzed byspectrophotometry. The levels of ALT were expressed as units per liter of supernatant (U/l). Lactate dehydrogenase (LDH) released into the medium solution from dead cells was measured by a LDH assay kit (cat. no. A020-2-2; Nanjing Jiancheng Institute of Biotechnology) according to the manufacturer's instructions and analyzed by spectrophotometry. The levels of LDH were expressed as units per liter of supernatant (U/l).

Cellular viability assay. The images of the cell morphology were captured using a light microscope (XDS-1A; Shanghai Precision Instrument Co., Ltd.). Cell viability was measured using a 3-(4,5-dimethylthiazol-2-yl)-2,5-diphenyltetrazolium bromide (MTT) assay (Sigma-Aldrich; Merck KGaA), according to the manufacturer's instructions. The MTT assays were quantified by reading the absorbance on a plate reader. The wavelength to measure the absorbance (Abs) of each sample was $492 \mathrm{~nm}$. MTT reduction measured at $492 \mathrm{~nm}$ was converted to percentage cell viability according to the following formula: \% cell viability=[(Abs $492 \mathrm{~nm}$ of treated group-blank)/(Abs $492 \mathrm{~nm}$ of control-blank)] x100.

Intracellular ROS assessment. Intracellular ROS levels were assessed using a ROSkit (cat. no. CA1410; Beijing Solarbio Science\&Technology Co., Ltd.). Dichlorodihydrofluorescein diacetate (DCFH-DA) was diluted $(1: 1,000)$ with serum-free medium to $10 \mu \mathrm{mol} / 1$. After the HCs were treated, the cells were washed and incubated with DCFH-DA for $20 \mathrm{~min}$ at $37^{\circ} \mathrm{C}$. Then the cells were washed three times before assessment. Fluorescence was detected and photographed with an inverted fluorescent microscope (magnification, x200; IX71; Olympus Corporation).

Reverse transcription-quantitative PCR (RT-qPCR). RT-qPCR was performed as previously described (17). Briefly, total RNA was extracted with the RNeasy mini kit (QiagenGmbH), according to the manufacturer's instructions. Then, complementary DNA (cDNA) was generated from $1 \mu \mathrm{g}$ of total RNA with $2 \mu \mathrm{M}$ oligo-dT primersand the Omniscript ${ }^{\mathrm{TM}}$ reverse transcriptase (both Qiagen GmbH). The iTaq SYBR-Green PCR Master Mix (Applied Biosystems; Thermo Fisher Scientific, Inc.) with specific primers for $\beta$-actin, IL-6, and TNF- $\alpha$ (Qiagen $\mathrm{GmbH}$ ) was used to perform qPCR. All samples were assayed in duplicate and normalized to the $\beta$-actin mRNA abundance. Gene expression levels were quantified using the $2^{-\Delta \Delta C q}$ method (22). The primers used for qPCR were the same as previously described (17).

Western blotting analysis. The western blotting protocol was the same as that previously described $(17,23)$. Briefly, HCs were collected in lysis buffer (Cell Signaling Technology, Inc.), sonicated, and centrifuged (16,000 x g for $\left.15 \mathrm{~min}, 4^{\circ} \mathrm{C}\right)$, after which the supernatant was collected. Protein concentrations were measured using a bicinchoninic acid protein assay kit (Thermo Fisher Scientific, Inc.). Samples were then run on SDS-PAGE and transferred to a polyvinylidene difluoride membrane. Then the membrane was incubated with primary and secondary antibodies, before being developed by an enhanced chemiluminescence kit (Thermo Fisher 
A
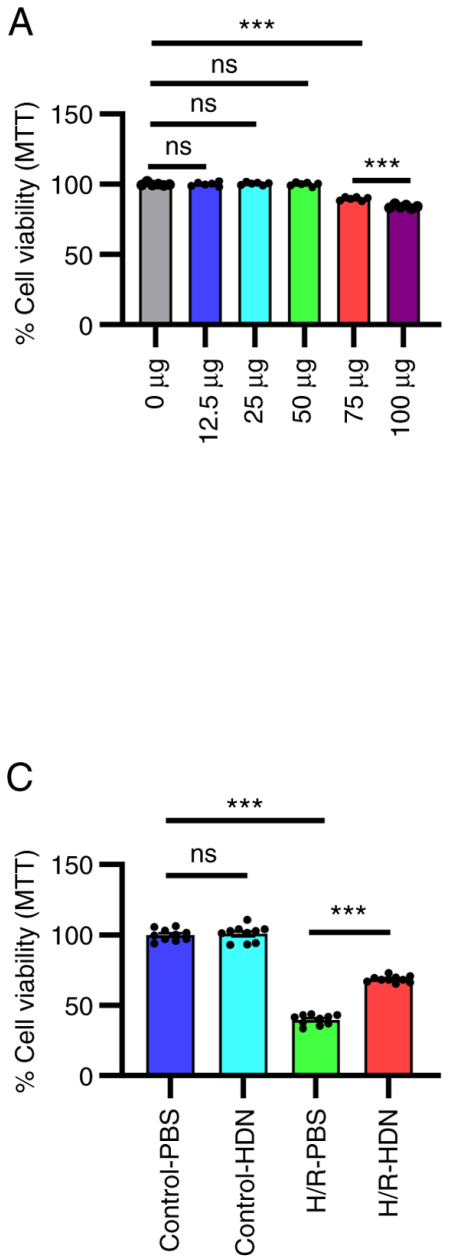

B
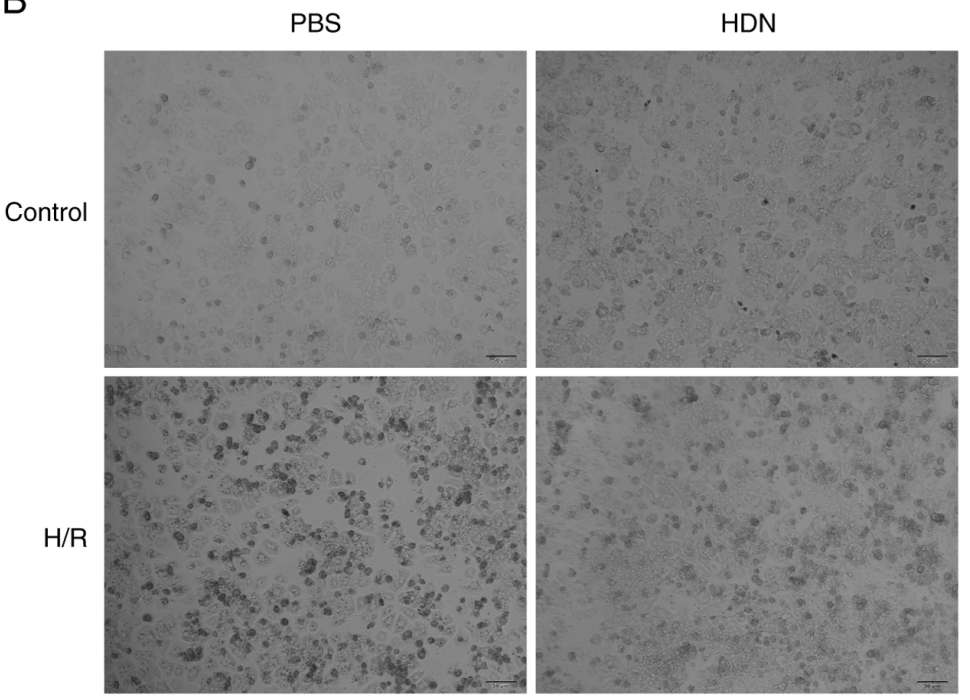

D

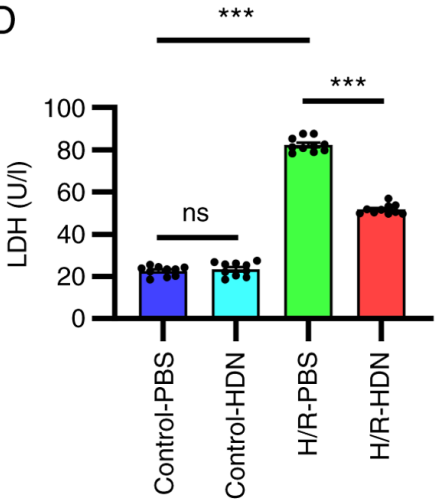

E

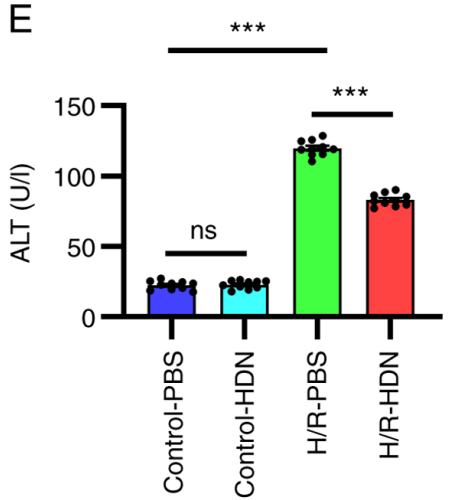

Figure 1. HDN protects HCs against H/R injury. (A) Cell viability was determined by MTT assay for HCs exposed to different doses of HDN under normoxic conditions. (B) Morphology of HCs in control PBS group, control HDN group, H/R PBS group and H/R HDN group under a light microscope (scale bar, $50 \mu \mathrm{m}$ ). (C) Cell viability was determined by MTT assayfor HCs in control PBS group, control HDN group, H/R PBS group and H/R HDN group. (D) Levels of supernatant LDH and (E) ALT in control PBS group, control HDN group, H/R PBS group and H/R HDN group. Results areshown as the mean \pm SEM of three independent experiments. ${ }^{* * *} \mathrm{P}<0.001$ with comparisons shown by lines. HDN, hesperidin; HC, hepatocyte; H/R, hypoxia/reoxygenation; LDH, lactate dehydrogenase; ALT, alanine aminotransferase; ns, not significant.

Scientific, Inc.). The signal was acquired with a ChemiDoc MP Imaging System (Bio-RadLaboratories, Inc.).

Autophagic flux. Autophagic flux was assessed as described previously (23). HCs were first cultured under hypoxic conditions, and then received reoxygenation with exposure to bafilomycin A1 (50 nM) treatment for $1 \mathrm{~h}$ (final hour of $8 \mathrm{~h}$ reoxygenation procedure). Western blotting was performed to analyse LC3 I: II conversion. In order to measure the accumulation of LC3 in HCs, the GFP-LC3 adenovirus was transfected into HCs before hypoxia, and then the HCs received the $\mathrm{H} / \mathrm{R}$ procedure involving bafilomycin A1 exposure. $\mathrm{HCs}$ transfected with GFP-LC3 were photographed with a Zeiss LSM510 laser-scanning confocal microscope (Carl Zeiss AG). GFP-LC3 puncta were counted with at least 5 green dots/cell from at least 30 cells/treatment.

\section{Results}

Hesperidin protects HCs against H/R injury. To determine the appropriate dose of HDN for HC treatment, HCs were isolated and exposed to different doses of HDN under normoxic conditions. As shown in Fig. 1A, there were significant side effects on cell viability with $\mathrm{HDN}$ doses $>50 \mu \mathrm{g} / \mathrm{ml}$. Therefore, the dose $50 \mu \mathrm{g} / \mathrm{ml}$ of HDN was used for subsequent experiments.

To determine the role of HDN in H/R-induced HC injury, $\mathrm{HCs}$ were isolated and cultured under $\mathrm{H} / \mathrm{R}$ conditions with or without HDN treatment. HC injury was evaluated by microscopic visualization of cell morphology and measured by an MTT assay and by detecting supernatant LDH and ALT levels. HCs appeared to be swelled into spherical shapes, and exhibited membrane rupture, nuclei pyknotic and nuclear condensation after H/R-induced injury under a light microscope (Fig. 1B). HDN treatment appeared to partially maintain HC cell morphology and numbers (Fig. 1B). The results of the MTT assay revealed that HC damage was significantly induced under H/R (Fig. 1C). As shown in Fig. 1B and C, HDN alone had no effect on HC morphology and cell viability. However, HDN treatment significantly ameliorated the cell viability under H/R conditions (Fig. 1C). Similarly, supernatant LDH levels and ALT levels increased significantly during $\mathrm{H} / \mathrm{R}$, and these levels were significantly reversed by HDN treatment (Fig. 1D and E). Taken together, 


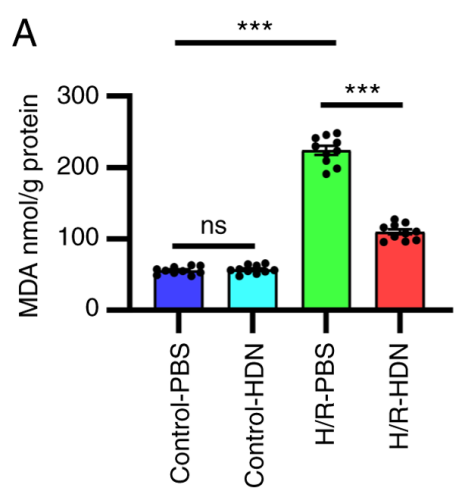

D
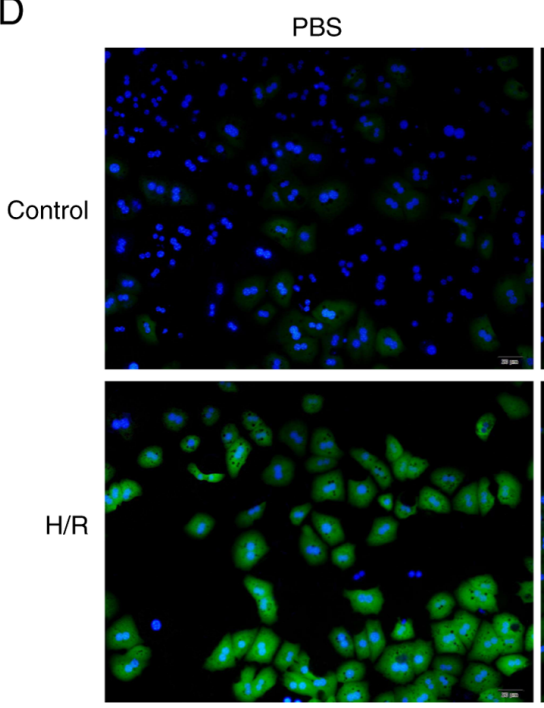

B
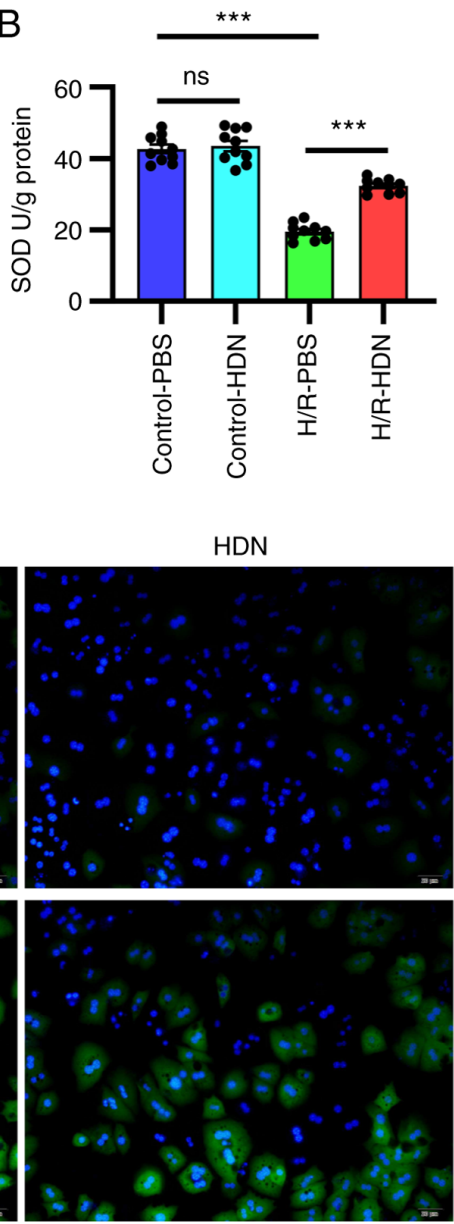

C
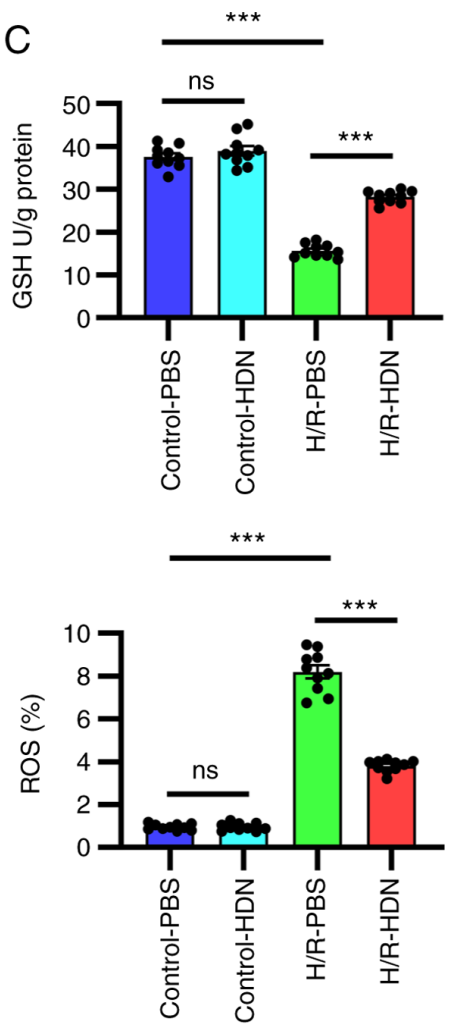

Figure 2. HDN attenuates hepatocyte oxidative stress induced by H/R. (A) MDA, (B) SOD, (C) GSH and (D) ROSlevels in control PBS group, control HDN group, H/R PBS group and H/R HDN group. ROS (green) was detected by fluorescence microscopy (magnification, $\mathrm{x} 40$ ). Results are shown as the mean \pm SEM of three independent experiments. ${ }^{* * *} \mathrm{P}<0.001$ with comparisons shown by lines. HDN, hesperidin; H/R, hypoxia/reoxygenation; MDA, malondialdehyde; SOD, superoxide dismutase; GSH, glutathione; ROS, reactive oxygen species; ns, not significant.

these data indicated that HDN protected HCs against H/R-induced injury.

Hesperidin attenuates $H C$ oxidative stress induced by $H / R$. The most characteristic mechanism of $\mathrm{H} / \mathrm{R}$-induced injury is oxidative stress $(3,8)$. HDN has been reported to attenuate oxidative stress in many situations, such as in I/R injury and toxin-induced damage $(17,24)$. To explore whether HDN attenuates oxidative stress during H/R, the levels of MDA, SOD, GSH and ROS were detected using commercial kits. Notably, compared with that of the control group, the MDA content increased significantly during H/R; however, this change was significantly reversed by exposure to HDN (Fig. 2A). By contrast, compared with that of the control group the antioxidant activities of SOD and GSH decreased significantly during $\mathrm{H} / \mathrm{R}$, and these effects were reversed by exposure to HDN (Fig. 2B and C). Similar findings were obtained using a fluorescent dye assay to detect the levels of ROS; H/R significantly induced ROS levels in HCs, while this was effectively reversed by HDN treatment (Fig. 2C). Taken together, these data indicated that HDN attenuated $\mathrm{HC}$ oxidative stress induced by $\mathrm{H} / \mathrm{R}$.

Hesperidin ameliorates HC inflammatory responses during $H / R$. The mechanisms of $H / R$-induced injury include both direct cellular damage resulting from oxidative stress and injury resulting from non-sterile inflammatory responses $(3,8)$. It has been reported that numerous cytokines are involved in H/R-induced injury (25-27). To ascertain the relationship between HDN and inflammation during H/R-induced injury, the levels of IL-6 and TNF- $\alpha$ were assessed by RT-qPCR and ELISA. The results demonstrated that, compared with those of the control group, the mRNA expression levels of IL- 6 and TNF- $\alpha$ in HCs and the concentrations of IL- 6 and TNF- $\alpha$ in the supernatants increased significantly following $H / R$, and HDN treatment significantly ameliorated these effects (Fig. 3). Thus, these data indicated that HDN ameliorated HC inflammatory responses during $\mathrm{H} / \mathrm{R}$.

Hesperidin induces autophagy to protect HCs against $H / R$ injury. Cell death is the end result of H/R-induced injury and can propagate injury through the activation of inflammatory pathways (25-27). It has been reported that autophagy protects against liver I/R injury in vivo and protects $\mathrm{HCs}$ against $\mathrm{H} / \mathrm{R}$ injury in vitro (23). We and others have demonstrated that $\mathrm{HDN}$ protects against I/R injury (13-17). To determine whether HDN may regulate protective autophagy during $\mathrm{H} / \mathrm{R}$ injury, the present study assessed the levels of specific autophagic markers in HCs with or without HDN exposure during H/R injury. Western blotting results 

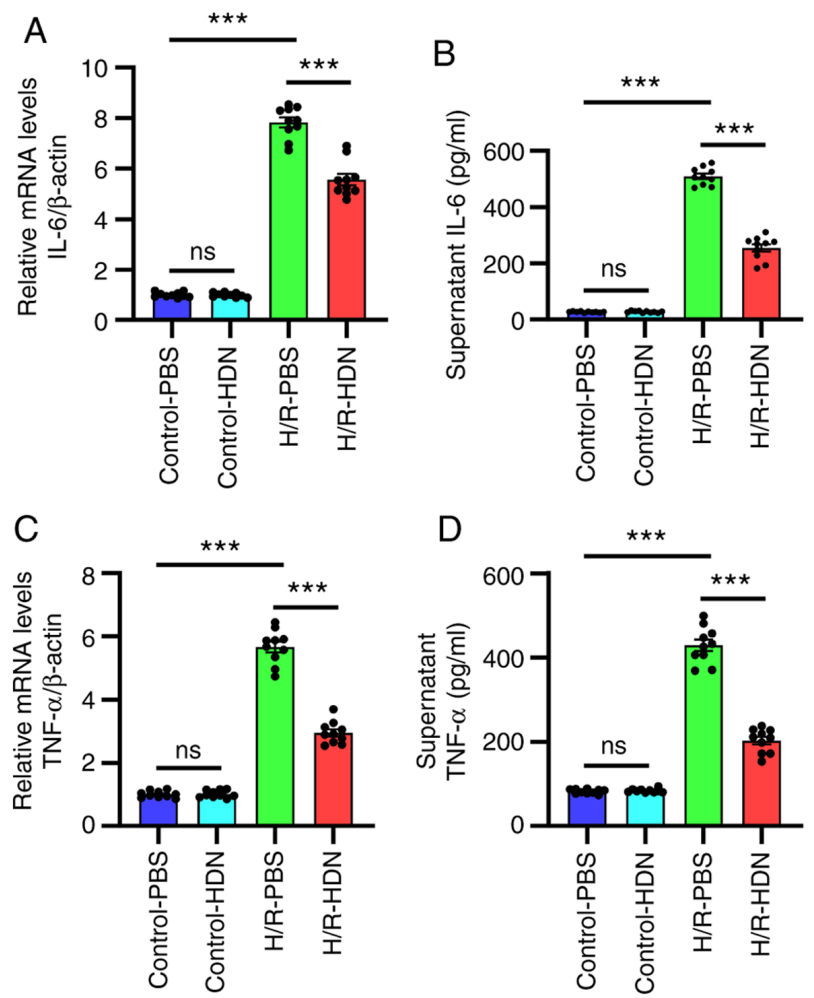

Figure 3. HDN ameliorates HC inflammatory responses during H/R . (A) mRNA expression levels of IL-6 in control PBS group, control HDN group, H/R PBS group and H/R HDN group. (B) Levels of IL-6 in supernatants assessed by ELISA. (C) mRNA expression levels of TNF- $\alpha$. (D) Levels of TNF- $\alpha$ in supernatants assessed by ELISA. Results are shown as the mean \pm SEM from three independent experiments. ${ }^{* * * *} \mathrm{P}<0.001$ with comparisons shown by lines. HDN, hesperidin; HC, hepatocyte $\mathrm{H} / \mathrm{R}$, hypoxia/reoxygenation; IL, interleukin; TNF, tumor necrosis factor; ns, not significant.

demonstrated that LC3-II and Beclin-1 protein expression levels increased, while P62 levels decreased during H/R upon exposure to HDN (Fig. 4A). It is important to know whether autophagosomes were formed from new autophagosome formation or from the blockade of autophagosome degradation during autophagy. To investigate these possibilities, HCs were cultured with or without HDN treatment under exposure to bafilomycin A1, which inhibits autophagolysosomal fusion and degradation, and then the $\mathrm{HCs}$ were subjected to normoxia or H/R. Western blotting results demonstrated that LC3-II levels increased in HCs with or without HDN treatment under the normoxia condition (Fig. 4B). However, LC3-II levels only increased in HCs exposed to HDN under the H/R conditions (Fig. 4C). In addition, GFP-LC3 was transfected into HCs and the accumulation of GFP-LC3 puncta was quantified by confocal microscopy. Consistent with the western blotting results, the numbers of GFP-LC3 puncta in HCs with exposure to HDN were greater compared with those observed in HCs without HDN exposure under the H/R conditions after bafilomycin A1 treatment (Fig. 4D). Taken together, these data indicated that HDN induced autophagy to protect HCs against $\mathrm{H} / \mathrm{R}$ injury.

\section{Discussion}

HDN is a flavonoid extracted from plants belonging to the families Lamiaceae and Betulaceae with a variety of bioactivities (9). It has been revealed that HDN has a variety of beneficial qualities, such as attenuating apoptosis, ameliorating hypotension, preventing hepatic steatosis, impairing dengue virus replication, and suppressing cancer proliferation (9-12). Additionally, it ameliorates I/R injury by attenuating oxidative stress and inflammation responses (17). However, whether HDN protects HCs against H/R-induced injury in vitro was largely unknown. The present study provided strong evidence that HDN protected HCs against H/R-induced injury by attenuating oxidative stress and ameliorating inflammatory responses while promoting autophagy. Thus, the current results identified HDN as a potential protective factor for HCs. These findings indicated that HDN may serve as a natural compound for ameliorating $\mathrm{HC}$ injury during $\mathrm{H} / \mathrm{R}$.

$\mathrm{I} / \mathrm{R}$ injury remains a critical challenge because it results in cell death and organ failure (28-30). Increasing evidence has revealed that the basic pathophysiology of $I / R$ is initiated from anaerobic metabolism and increasing ROS production $(31,32)$. The dramatically increased content of ROS is due to excessive production of ROS and lower levels of antioxidant factors $(33,34)$. Thus, how to balance the oxidative stress is a crucial point in dealing with I/R injury. Increasing studies have revealed that HDN possesses an antioxidant effect (9). The present studydemonstrated that the MDA content and ROS levels increased during $\mathrm{H} / \mathrm{R}$ and were significantly reversed following exposure to HDN. However, the antioxidant indicators SOD and GSH decreased during H/R and were reversed following exposure to HDN. Taken together, these results suggested that HDN attenuated $\mathrm{HC}$ oxidative stress induced by $\mathrm{H} / \mathrm{R}$, which is consistent with previous studies $(19,35,36)$. Ebegboni et al (19) found that flavonoids were associated with ROS modulation, reducing the generation of superoxide/hydrogen peroxide during H/R-induced oxidative stress in a human first-trimester trophoblast cell line. Chen et al (35) observed that hesperitin (an active metabolite of HDN) significantly attenuated oxidative stress-induced apoptosis by reducing ROS levels in cisplatin-treated HK-2 cells in vitro. In a sodium arsenite (SA)-induced nephrotoxicity and hepatotoxicity model, Turk et al (36) observed that HDN co-treatment had an antioxidant effect on SA-induced toxicity and aided in protecting the tissue architecture by decreasing MDA and 8-hydroxy-2'-deoxyguanosine levels and increasing the GSH level and SOD, catalase (CAT), and glutathione peroxidase (GPx) activities. However, the mechanism by which HDN regulates oxidative stress remains unknown.

Anti-inflammatory effects are one of the most important effect types of HDN. Excessive inflammatory responses are supposed to be the major mechanism of I/R injury in the later phase (8). Regulating inflammatory responses provides a novel therapeutic and preventive target for I/R injury. The present study found that the mRNA expression levels of IL-6 and TNF- $\alpha$ in HCs and the concentrations of IL- 6 and TNF- $\alpha$ in the supernatants increased significantly after $H / R$, and HDN significantly ameliorated these effects. These results suggested that HDN may ameliorate inflammation in H/R. We and others have confirmed that HDN attenuates inflammatory responses in I/R injury $(9,17)$. In a chronic unpredictable mild stress (CUMS)-induced rat model, Xie et al (37) found that HDN treatment significantly relieved depressive-like behaviors in CUMS rats by decreasing the expression levels 


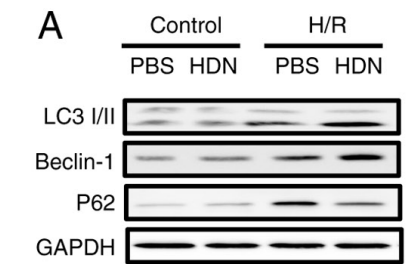

B

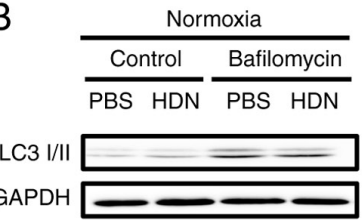

C

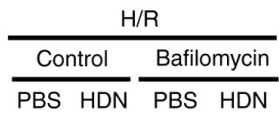

LC3 I/II $=$

GAPD

$\mathrm{H} / \mathrm{R}$

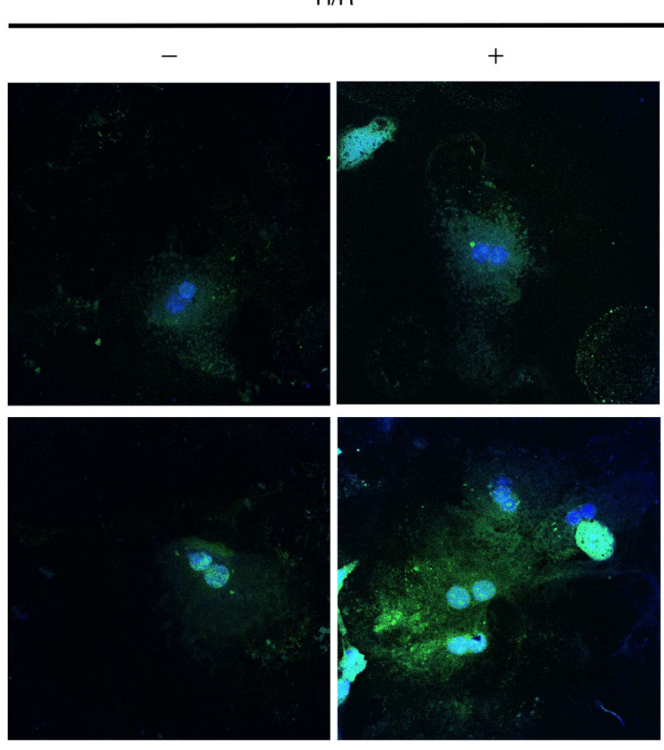

D
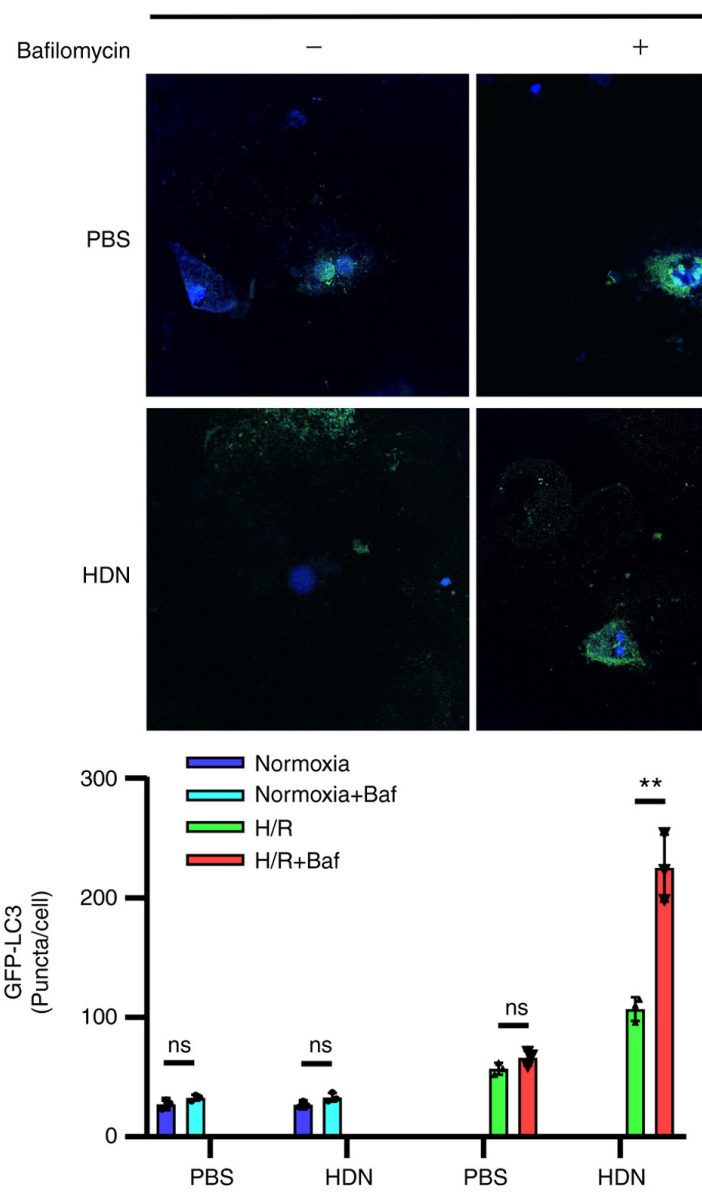

$+$
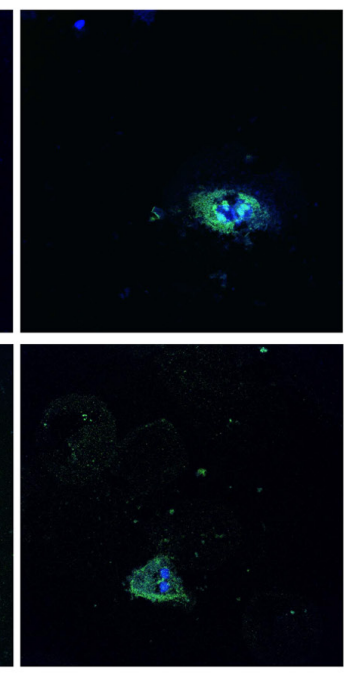

Figure 4. HDN induces autophagy to protect HCs against H/R injury. (A) Representative western blots showing the protein expression levels of LC3, Beclinand P62 in control PBS group, control HDN group, H/R PBS group and H/R HDN group. (B) Representative western blots showing the levels of LC3 in HCs subjected to normoxia with or without bafilomycin A1 $(50 \mathrm{nM})$. (C) Representative western blots showing the levels of LC3 in HCs subjected to H/R with or without bafilomycin A1 (50 nM). (D) Confocal microscopy images of HCs overexpressing GFP-LC3 (green) and subjected to normoxia or H/R, with/without bafilomycin A1 (50 nM) treatment (magnification, x40). Nuclei were counterstained with Hoechst 33342 (blue). The numbers of GFP-LC3 puncta were counted per cell and represented in the bar graph. Results are shown as the mean \pm SEM of three independent experiments. ${ }^{* *} \mathrm{P}<0.01$ with comparisons shown by lines. HDN, hesperidin; HC, hepatocyte; H/R, hypoxia/reoxygenation; LC3, microtubule-associated protein 1 light chain $3 \alpha$; P62, sequestosome 1; ns, not significant.

of IL-6 and TNF- $\alpha$ in the prefrontal cortex and microglia. In addition, they found that the relative mechanisms were based on the NLR family pyrin domain containing 3 inflammatory signaling pathway (37). Heo et al (38) revealed that HDN treatment in rats with spinal cord injury reduced neuropathological changes (includinghemorrhage, inflammatory cell infiltration, and tissue loss) and levels of proinflammatory cytokines, such as TNF- $\alpha$. Collectively, these data imply that HDN ameliorates inflammatory responses to protect against tissue injury.

Autophagy is required in diverse paradigms of lifespan extension, leading to the prevailing notion that autophagy can maintain cell homeostasis and ensure cell survival under stressful conditions $(39,40)$. We and others have revealed that autophagy has a pivotal role in maintaining cell survival following liver I/R injury $(23,41)$. The present study found that the protein levels of autophagy makers LC3-II and Beclin-1 increased, while P62 levels decreased during H/R under exposure to HDN. Under exposure to bafilomycin A1, which inhibits autophagolysosomal fusion and degradation, LC3-II levels increased in HCs exposed to HDN under the H/R conditions. Furthermore, the results were confirmed by microscopic evaluation of GFP-LC3 puncta in HCs through confocal microscopy. Taken together, these results suggested that HDN induced autophagy to protect $\mathrm{HCs}$ against $\mathrm{H} / \mathrm{R}$ injury. In a previous study, Saiprasad et al (42) found that HDN supplementation initiated apoptosis via targeted inhibition of constitutively activated Aurora-A-mediated PI3K/Akt/glycogen synthase kinase-3 $\beta$ and mTOR pathways coupled with autophagic stimulation against azoxymethane-induced colon carcinogenesis. 


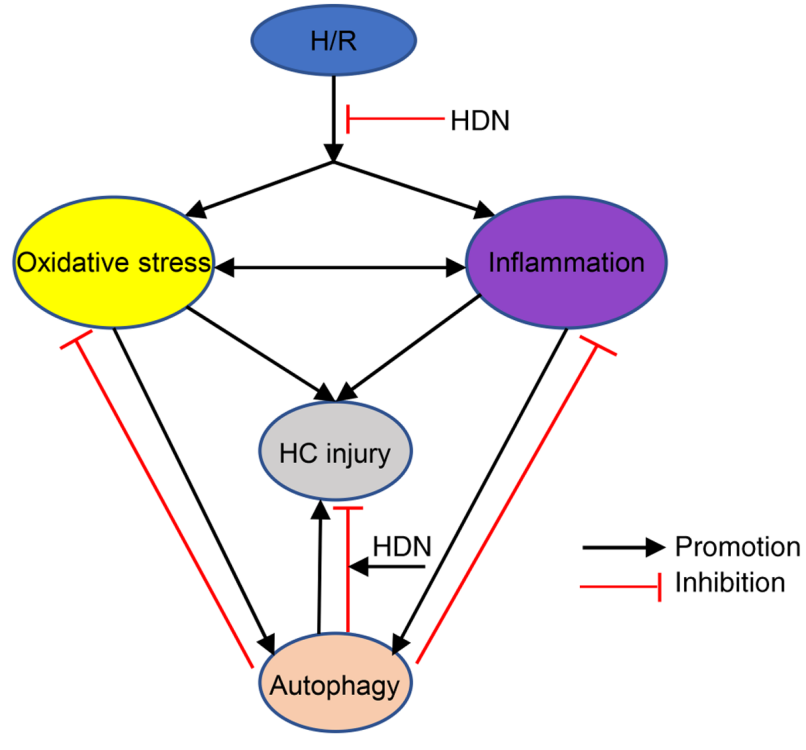

Figure 5. Schematic demonstrating the interplay among oxidative stress, inflammatory activity, and autophagy following H/R injury. H/R leads to oxidative stress, inflammatory activity, and autophagy, while HDN exposure inhibits these effects. Both oxidative stress and inflammation induce autophagy and $\mathrm{HC}$ injury. Appropriate autophagy activity contributes to $\mathrm{HC}$ recovery by reducing oxidative stress and inflammatory activity, while autophagy dysfunction aggravates HC injury. HDN exposure promotes the effects of autophagy, thus reducing HCinjury. H/R, hypoxia/reoxygenation; HDN, hesperidin; HC, hepatocyte.

Zhu et al (43) revealed that autophagic activation may be involved in the mechanism of hesperidin's therapeutic effects on cognitive impairment. However, Li et al (15) found that excessive autophagy exacerbates myocardial I/R injury and that HDN could reduce myocardial I/R injury by suppressing excessive autophagy. These opposing observations deserve further study and discussion. In a previous study, Turk et al (36) found that HDN could decrease oxidative stress, inhibit inflammation and reduce apoptosis to protect against SA-induced liver toxicity. The present study revealed that HDN attenuated oxidative stress, inhibited the production of pro-inflammatory cytokines and induced autophagy to protect hepatocytes against H/R-induced injury. Induction of autophagy may be the underlying mechanism by which HDN aids in hepatoprotection.

As aforementioned, the effects of HDN on autophagy appear to be controversial. In the present study, cultured HCs were injured by H/R stimulation, presumably by oxidative stress. HDN exposure rescued the damaging effects of H/R stimulation. Additionally, exposure toHDNincreased autophagy in H/R-treated HCs, as shown in Fig. 4. It would be interesting to know how much death in HCs after H/R treatment was due to autophagy and how much was due to oxidative injury, however this is difficult to determine.It appears that HDN exposure could reduce oxidative stress levels by approximately half, according to Fig. 2. It has been reported that oxidative stress and inflammatory activity are two early events in the cascade of I/R injury and H/R injury $(41,44)$. These two factors also directly trigger the development of autophagy $(41,44)$. Taken together, it can be assumed that appropriate autophagy activity contributes to HC recovery by reducing oxidative stress and inflammatory activity, while autophagy dysfuntion aggravates HC injury (Fig. 5). The detailed mechanisms by which HDN regulates oxidative stress, inflammatory activity and autophagy balance deserve further study in the future.

In summary, the present study demonstrated that HDN attenuated $\mathrm{HC}$ oxidative stress and inflammatory responses while enhancing autophagy during H/R. HDN may have a protective effect on HCs during H/R-induced injury. These findings broaden our understanding of the functions of HDN in acute sterile inflammation and raise the possibility that HDN may be used to protect organs against I/R injury.

\section{Acknowledgements}

Not applicable.

\section{Funding}

This study was supported by The Young Teachers' Basic Ability Improvement in Guangxi University Project (grant no. 2019KY0108), the National Natural Science Foundation of China (grant no. 81960358),Talents Sub-Highland of Emergency and Medical Rescue of Guangxi Province in China (grant no. GXJZ201405), Health Commission of Guangxi (grant no. Z2016289), the Guangxi Natural Science Foundation (grant nos. 2018JJB140279 and 2020GXNSFAA159127), Guangxi Key Laboratory for the Prevention and Control of Viral Hepatitis (grant no. GXCDCKL201902) and the Medical Excellence Award funded by the Creative Research Development Grant from the First Affiliated Hospital of Guangxi Medical University (grant no. 2017-11-7).

\section{Availability of data and materials}

The datasets used and /or analyzed during the current study are available from the corresponding authors on reasonable request.

\section{Authors' contributions}

SL and JZ designed and performed the majority of experiments, isolated and cultured hepatocytes, collected and analyzed the experimental data, and drafted the manuscript. LP, QQ and DL performed the supernatant analysis, MTT assay and ELISA. PW, WP and YW performed autophagic flux and conducted the immunofluorescence staining. YX performed the RT-qPCR and western blot analysis. LS and XY supervised the project, provided technical advice, conducted analyses of the raw data, and reviewed and edited the manuscript.SL and XY confirm the authenticity of all the raw data. All authors read and approved the final manuscript.

\section{Ethics approval and consent to participate}

Protocols involving animals were approved by The Animal Care and Use Committee of The First Affiliated Hospital of Guangxi Medical University (Nanning, China), and the experiments were performed in adherence to the National Institutes of Health guidelines for the use of laboratory animals. 


\section{Patient consent for publication}

Not applicable.

\section{Competing interests}

The authors declare that they have no competing interests.

\section{References}

1. Nastos C, Kalimeris K, Papoutsidakis N, Tasoulis MK Lykoudis PM, Theodoraki K, Nastou D, Smyrniotis V and Arkadopoulos N: Global consequences of liver ischemia/reperfusion injury. Oxid Med Cell Longev 2014: 906965 2014.

2. Schemmer P,Lemasters JJ and Clavien PA: Ischemia/Reperfusion injury in liver surgery and transplantation. HPB Surg 2012: 453295, 2012.

3. Sinning C, Westermann D and Clemmensen P: Oxidative stress in ischemia and reperfusion: Current concepts, novel ideas and future perspectives. Biomark Med 11: 11031-11040, 2017.

4. Halladin NL: Oxidative and inflammatory biomarkers of ischemia and reperfusion injuries. Dan Med J 62: B5054, 2015

5. Tan S, Yokoyama Y, Wang Z, Zhou F, Nielsen V, Murdoch AD, Adams $\mathrm{C}$ and Parks DA: Hypoxia-Reoxygenation is as damaging as ischemia-reperfusion in the rat liver. Crit Care Med 26: 1089-1095, 1998.

6. Portal L, Martin V, Assaly R, de Tassigny A, Michineau S, Berdeaux A, Ghaleh B and Pons S: A model of hypoxia-reoxygenation on isolated adult mouse cardiomyocytes: Characterization, comparison with ischemia-reperfusion, and application to the cardioprotective effect of regular treadmill exercise. J Cardiovasc Pharmacol Ther 18: 367-375, 2013.

7. Zou X, Liu Q, Guo S, Zhu J, Han J, Xia Z, Du Y, Wei L and Shang J: A novel zebrafish larvae hypoxia/reoxygenation model for assessing myocardial ischemia/reperfusion injury. Zebrafish 16: 434-442, 2019.

8. Jimenez-Castro MB, Cornide-Petronio ME, Gracia-Sancho J and Peralta C: Inflammasome-Mediated inflammation in liver ischemia-reperfusion injury. Cells 8: 1131, 2019.

9. Parhiz H, Roohbakhsh A, Soltani F, Rezaee R and Iranshahi M: Antioxidant and anti-inflammatory properties of the citrus flavonoids hesperidin and hesperetin: An updated review of their molecular mechanisms and experimental models. Phytother Res 29: 323-331, 2015

10. Elhelaly AE, AlBasher G, Alfarraj S, Almeer R, Bahbah EI, Fouda MMA, Bungau SG, Aleya L and Abdel-Daim MM: Protective effects of hesperidin and diosmin against acrylamide-induced liver, kidney, and brain oxidative damage in rats. Environ Sci Pollut Res Int 26: 35151-35162, 2019.

11. Pandey P, Sayyed U, Tiwari RK, Siddiqui MH, Pathak N and Bajpai P: Hesperidin induces ROS-mediated apoptosis along with cell cycle arrest at $\mathrm{G} 2 / \mathrm{M}$ phase in human gall bladder carcinoma. Nutr Cancer 71: 676-687, 2019.

12. Mo'men YS, Hussein RM and Kandeil MA: Involvement of PI3K/Akt pathway in the protective effect of hesperidin against a chemically induced liver cancer in rats. J Biochem Mol Toxicol 33: e22305, 2019.

13. Park HK, Kang SW and Park MS: Hesperidin ameliorates hepatic ischemia-reperfusion injury in sprague-dawley rats. Transplant Proc 51: 2828-2832, 2019

14. Park WS, Park MS, Kang SW, Jin SA, Jeon Y, Hwang J and Kim SK: Hesperidin shows protective effects on renal function in ischemia-induced acute kidney injury (Sprague-Dawley Rats) Transplant Proc 51: 2838-2841, 2019.

15. Li X, Hu X, Wang J, Xu W, Yi C, Ma R and Jiang H: Inhibition of autophagy via activation of PI3K/Akt/mTOR pathway contributes to the protection of hesperidin against myocardial ischemia/reperfusion injury. Int J Mol Med 42: 1917-1924, 2018.

16. Meng X, Wei M, Wang D, Qu X, Zhang K, Zhang N and Li X: The protective effect of hesperidin against renal ischemia-reperfusion injury involves the TLR-4/NF- $\mathrm{B} / \mathrm{iNOS}$ pathway in rats. Physiol Int 107: 82-91, 2020.

17. Li S, Qin Q, Luo D, Pan W, Wei Y, Xu Y, Zhu J and Shang L: Hesperidin ameliorates liver ischemia/reperfusion injury via activation of the akt pathway. Mol Med Rep 22: 4519-4530, 2020
18. He S, Wang X, Zhong Y, Tang L, Zhang Y, Ling Y, Tan Z, Yang $\mathrm{P}$ and Chen A: Hesperetin post-treatment prevents rat cardiomyocytes from hypoxia/reoxygenation injury in vitro via activating PI3K/Akt signaling pathway. Biomed Pharmacother 91: 1106-1112, 2017.

19. Ebegboni VJ, Dickenson JM and Sivasubramaniam SD: Antioxidative effects of flavonoids and their metabolites against hypoxia/reoxygenation-induced oxidative stress in a human first trimester trophoblast cell line. Food Chem 272: 117-125, 2019.

20. Lei Z, Deng M, Yi Z, Sun Q, Shapiro RA, Xu H, Li T, Loughran PA, Griepentrog JE, Huang H, et al: cGAS-mediated autophagy protects the liver from ischemia-reperfusion injury independently of STING. Am J Physiol Gastrointest Liver Physiol 314: G655-G667, 2018

21. Sun Q, Loughran P, Shapiro R, Shrivastava IH, Antoine DJ, Li T, Yan Z, Fan J, Billiar TR and Scott MJ: Redox-Dependent regulation of hepatocyte absent in melanoma 2 inflammasome activation in sterile liver injury in mice. Hepatology 65: 253-268, 2017.

22. Livak KJ and Schmittgen TD: Analysis of relative gene expression data using real-time quantitative PCR and the 2(-Delta Delta C(T)) method. Methods 25: 402-408, 2001.

23. Li S, Yi Z, Deng M, Scott MJ, Yang C, Li W, Lei Z, Santerre NM, Loughran P and Billiar TR: TSLP protects against liver I/R injury via activation of the PI3K/Akt pathway. JCI Insight 4: e129013, 2019

24. Iranshahi M, Rezaee R, Parhiz H, Roohbakhsh A and Soltani F: Protective effects of flavonoids against microbes and toxins: The cases of hesperidin and hesperetin. Life Sci 137: 125-132, 2015.

25. Cernanec J, Guilak F, Weinberg JB, Pisetsky DS and Fermor B: Influence of hypoxia and reoxygenation on cytokine-induced production of proinflammatory mediators in articular cartilage. Arthritis Rheum 46: 968-975, 2002.

26. Cheng Y, Xiong J, Chen Q, Xia J, Zhang Y, Yang X, Tao K, Zhang $\mathrm{S}$ and He S: Hypoxia/reoxygenation-induced HMGB1 translocation and release promotes islet proinflammatory cytokine production and early islet graft failure through TLRs signaling. Biochim Biophys Acta Mol Basis Dis 1863: 354-364, 2017.

27. Chenevier-Gobeaux C, Simonneau C, Lemarechal $\mathrm{H}$, Bonnefont-Rousselot D, Poiraudeau S, Rannou F, Ekindjian OG, Anract P and Borderie D: Effect of hypoxia/reoxygenation on the cytokine-induced production of nitric oxide and superoxide anion in cultured osteoarthritic synoviocytes. Osteoarthritis Cartilage 21: 874-881, 2013.

28. Fernandez AR, Sánchez-Tarjuelo R, Cravedi P, Ochando J and López-Hoyos M: Review: Ischemia reperfusion injury-A translational perspective in organ transplantation. Int J Mol Sci 21: $8549,2020$.

29. Arriel RA, Rodrigues JF, Souza HLR, Meireles A, Leitao LFM, Crisafulli A and Marocolo M: Ischemia-Reperfusion intervention: From enhancements in exercise performance to accelerated performance recovery-a systematic review and meta-analysis. Int J Environ Res Public Health 17: 8161, 2020

30. Nieuwenhuijs-Moeke GJ, Pischke SE, Berger SP, Sanders JSF, Pol RA, Struys M, Ploeg RJ and Leuvenink HGD: Ischemia and reperfusion injury in kidney transplantation: Relevant mechanisms in injury and repair. J Clin Med 9: 253, 2020.

31. Yi Z, Deng M, Scott MJ, Fu G, Loughran PA, Lei Z, Li S, Sun P, Yang C, Li W, et al: Immune-Responsive gene 1/itaconate activates nuclear factor erythroid 2-related factor 2 in hepatocytes to protect against liver ischemia-reperfusion injury. Hepatology 72 : 1394-1411, 2020

32. Guan LY, Fu PY, Li PD, Li ZN, Liu HY, Xin MG and Li W: Mechanisms of hepatic ischemia-reperfusion injury and protective effects of nitric oxide. World J Gastrointest Surg 6: 122-128, 2014.

33. Nita M and Grzybowski A: The role of the reactive oxygen species and oxidative stress in the pathomechanism of the age-related ocular diseases and other pathologies of the anterior and posterior eye segments in adults. Oxid Med Cell Longev 2016: 3164734, 2016.

34. He L, He T, Farrar S, Ji L, Liu T and Ma X: Antioxidants maintain cellular redox homeostasis by elimination of reactive oxygen species. Cell Physiol Biochem 44: 532-553, 2017.

35. Chen X, Wei W, Li Y, Huang J and Ci X: Hesperetin relieves cisplatin-induced acute kidney injury by mitigating oxidative stress, inflammation and apoptosis. Chem Biol Interact 308: 269-278, 2019. 
36. Turk E, Kandemir FM, Yildirim S, Caglayan C, Kucukler S and Kuzu M: Protective effect of hesperidin on sodium arsenite-induced nephrotoxicity and hepatotoxicity in rats. Biol Trace Elem Res 189: 95-108, 2019.

37. Xie L, Gu Z, Liu H, Jia B, Wang Y, Cao M, Song R, Zhang Z and Bian Y: The anti-depressive effects of hesperidin and the relative mechanisms based on the NLRP3 inflammatory signaling pathway. Front Pharmacol 11: 1251, 2020.

38. Heo SD, Kim J, Choi Y, Ekanayake P, Ahn M and Shin T: Hesperidin improves motor disability in rat spinal cord injury through anti-inflammatory and antioxidant mechanism via Nrf-2/HO-1 pathway. Neurosci Lett 715: 134619, 2020.

39. Zhou B, Kreuzer J, Kumsta C, Wu L, Kamer KJ, Cedillo L, Zhang Y, Li S, Kacergis MC, Webster CM, et al: Mitochondrial permeability uncouples elevated autophagy and lifespan extension. Cell 177: 299-314, 2019.

40. Wang K: Autophagy and apoptosis in liver injury. Cell Cycle 14: $1631-1642,2015$

41. Li J, Lin W and Zhuang L: CD5L-Induced activation of autophagy is associated with hepatoprotection in ischemic reperfusion injury via the CD36/ATG7 axis. Exp Ther Med 19: 2588-2596, 2020.
42. SaiprasadG,ChitraP,ManikandanR andSudhandiran G:Hesperidin induces apoptosis and triggers autophagic markers through inhibition of Aurora-A mediated phosphoinositide-3-kinase/Akt/mammalian target of rapamycin and glycogen synthase kinase- 3 beta signalling cascades in experimental colon carcinogenesis. Eur J Cancer 50: 2489-2507, 2014

43. Zhu B, Yang C, Wu J and Hua F: Autophagic activation may be involved in the mechanism of hesperidin's therapeutic effects on cognitive impairment. J Neurol Sci 351: 202-203, 2015.

44. He J, Liu J, Huang Y, Tang X, Xiao H and Hu Z: Oxidative stress, inflammation, and autophagy: Potential targets of mesenchymal stem cells-based therapies in ischemic stroke. Front Neurosci 15: 641157, 2021

cc (7) (9) This work is licensed under a Creative Commons (c) $\mathrm{EY}$ No No Attribution-NonCommercial-NoDerivatives 4.0 International (CC BY-NC-ND 4.0) License. 